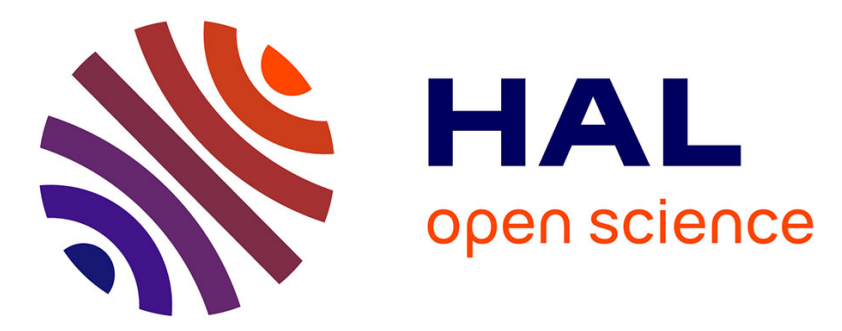

\title{
Probing pH-Responsive Interactions between Polymer Brushes and Hydrogels by Neutron Reflectivity
}

Guillaume Sudre, Dominique Hourdet, Costantino Creton, Fabrice Cousin, Yvette Tran

\section{- To cite this version:}

Guillaume Sudre, Dominique Hourdet, Costantino Creton, Fabrice Cousin, Yvette Tran. Probing pHResponsive Interactions between Polymer Brushes and Hydrogels by Neutron Reflectivity. Langmuir, 2014, 30 (32), pp.9700-9706. 10.1021/la501568p . hal-01087952

\section{HAL Id: hal-01087952 https://hal.science/hal-01087952}

Submitted on 9 May 2019

HAL is a multi-disciplinary open access archive for the deposit and dissemination of scientific research documents, whether they are published or not. The documents may come from teaching and research institutions in France or abroad, or from public or private research centers.
L'archive ouverte pluridisciplinaire HAL, est destinée au dépôt et à la diffusion de documents scientifiques de niveau recherche, publiés ou non, émanant des établissements d'enseignement et de recherche français ou étrangers, des laboratoires publics ou privés. 


\section{Probing pH-Responsive Interactions between}

\section{Polymer Brushes and Hydrogels by}

\section{Neutron Reflectivity}

Guillaume Sudre,,${ }^{\mathrm{a}, \mathrm{b}, ~ *}$ Dominique Hourdet, ${ }^{\mathrm{a}}$ Costantino Creton, ${ }^{\mathrm{a}}$ Fabrice Cousin, ${ }^{\mathrm{c}}$ Yvette $\operatorname{Tran}^{\mathrm{a}}$

${ }^{a}$ Laboratoire de Science et Ingénierie de la Matière Molle, UMR 7615 CNRS/UPMC/ESPCI ParisTech, 10 rue Vauquelin, F-75231 Paris Cedex 5, France

${ }^{b}$ Université de Lyon, Univ Lyon 1, CNRS, Ingénierie des Matériaux Polymères (IMP - UMR 5223), 15 Bd A. Latarjet, 69622 Villeurbanne Cedex, France.

${ }^{c}$ Laboratoire Léon Brillouin, CEA-CNRS, Saclay 91191 Gif-sur-Yvette Cedex, France

* Corresponding author: G. Sudre (e-mail: guillaume.sudre@univ-lyon1.fr)

Published in Langmuir:

Probing pH-Responsive Interactions between Polymer Brushes and Hydrogels by Neutron Reflectivity, Sudre G., Hourdet D., Creton C., Cousin F., Tran Y., Langmuir, 2014, 30(32), 9700-9706.

Link to the publisher version: http://dx.doi.org/10.1021/la501568p

Creative Commons Attribution Non-Commercial No Derivatives License 


\begin{abstract}
We investigated the effect of specific interactions on the structure of interfaces between a brush and a hydrogel, at the polymer chain length scale. We used a model system for which the interactions between the brush and the gel are switchable. We synthesized weak polyelectrolyte brushes of poly(acrylic acid) and hydrogels of polyacrylamide and $\operatorname{poly}(N, N$ dimethylacrylamide) which interact solely when the poly(acrylic acid) is mainly in its acidic form. The monomer density profiles of the poly(acrylic acid) brush immersed in pure deuterium oxide $\left(\mathrm{D}_{2} \mathrm{O}\right)$ or in contact with a $\mathrm{D}_{2} \mathrm{O}$-swollen gel were determined by neutron reflectivity. At $\mathrm{pH} 2$ when the brush is in its neutral and acid form, it interacts with the gel by hydrogen bonds while at $\mathrm{pH} 9$ when the brush is a polyelectrolyte, it is not interacting with the gel. Our results show that the presence of interactions with the gel at $\mathrm{pH} 2$ increases the swelling ratio of the brush relative to pure $\mathrm{D}_{2} \mathrm{O}$, meaning that the brushes exhibit conformations which are more extended from the surface than in the absence of interactions.
\end{abstract}




\section{INTRODUCTION}

Many hydrogels, in particular those originating from renewable resources, are used in food science, biology and biomedicine or targeted drug delivery or artificial tissue applications. ${ }^{1-5}$ Hydrogels are attractive biomaterials, generally biocompatible, highly deformable and with tunable mechanical properties through their chemistry. However, since they contain large amounts of water, they are also fragile and not naturally sticky. ${ }^{6}$ Yet, their adhesive or lubrication properties are essential in many applications. ${ }^{7,8}$

The use of simple model systems should lead to a better understanding of the mechanisms controlling the adhesion between hydrogels and surfaces. To control and tune the adhesive properties of hydrogels on surfaces, two approaches can be considered: the functionalization of hydrogels with responsive chemical groups which can then trigger interactions with a substrate or the modification of the surface with responsive polymers which can interact with the hydrogel network. ${ }^{9}, 10$ We chose the second approach and used responsive polymer brushes synthesized by chemical grafting onto solid substrates for their ease of synthesis and ease of verification of successful synthesis. ${ }^{11}$ Polymer brushes have been widely used for that reason to functionalize surfaces and to control the adsorption properties ${ }^{12}$ of bacteria, ${ }^{13}$ cells, ${ }^{14}$ antibodies, ${ }^{15,16}$ surfactants ${ }^{17}$, nanoparticles ${ }^{18}$ or proteins. ${ }^{19-24}$

In the studies performed with elastomers in the absence of solvent, surface tethered polymer chains can greatly enhance the adhesion phenomena between the functionalized surface and an elastomeric network: when the surface of the elastomer is in contact with the grafted surface, the entanglements between the brush chains and the elastomer are the cause of a

significant increase in adhesion. ${ }^{25}$ However, in the case of diluted networks such as hydrogels, the effect of entanglements should be substantially reduced and only specific molecular interactions between monomer units should contribute to adhesion. 
We selected chemically cross-linked neutral model hydrogels and brushes which are $\mathrm{pH}$ sensitive. The molecular interactions between the hydrogel and the polymer brush in water are hydrogen bonds triggered by a change in $\mathrm{pH}^{26}$ In acidic conditions $(\mathrm{pH}<3)$, interpolymer complexes between the proton-donor poly(acrylic acid) and the proton-acceptor poly(acrylamide) (PAM) or poly $\left(N, N\right.$-dimethylacrylamide) (PDMA) are expected to occur. ${ }^{27}$ The pH-responsive PAA brushes were synthesized on silicon wafers by the "grafting onto" $\operatorname{approach}^{28}$ and hydrogels of PAM or PDMA were prepared by redox free radical polymerization. The gels were designed for the adhesion tests: highly elastic ( $\left.G^{\prime}>>G^{\prime \prime}\right)$ and dimensionally stable upon $\mathrm{pH}$, ionic strength and temperature changes. The adhesive properties between the neutral gels and the $\mathrm{pH}$-responsive brushes can then be tuned simply by a change in $\mathrm{pH}$ without any change in mechanical or structural characteristics of the gel. Besides the macroscopic approach consisting in underwater adhesion tests, ${ }^{26}$ the effect of the interactions between the hydrogel and the polymer brush can be probed at the molecular scale by neutron reflectivity which gives access to the profile of neutron scattering density across a planar interface. We previously determined the monomer volume fraction as a function of distance from the surface for PAA brushes at various $\mathrm{pH}^{29}$ Here, we probe the interface between a hydrogenated polymer brush and a hydrogenated gel swollen in deuterium oxide. Using poly(N-isopropylacrylamide) (PNIPAM) brushes as a control sample, we show that the swollen hydrogel can be considered a continuous medium. Then, the reflectivity data can be used to determine the monomer concentration profile of the PAA brushes. We focus on how the structure of the brush, in contact with the swollen hydrogel, changes with the variation of the specific interactions between the monomers of the brush and those of the gel. 


\section{EXPERIMENTAL SECTION}

\subsection{Synthesis of PAA and PNIPAM brushes}

The synthesis of poly(acrylic acid) brushes on silicon wafers using the "grafting onto" strategy was reported elsewhere. ${ }^{28}$ Briefly, the carboxylic acid functions of carboxyterminated poly(tert-butylacrylate) chains were thermally reacted at $120^{\circ} \mathrm{C}$ on a silicon single crystal previously functionalized with a self-assembled monolayer of $\gamma$ glycidoxypropyltrimethoxysilane, (GPS). Then the ester functions were degraded by pyrolisis at $200{ }^{\circ} \mathrm{C}$ to obtain PAA brushes (either short chains of $M=2.4 \mathrm{~kg}^{\mathrm{mol}}{ }^{-1}$, or long chains of $M$ $=24 \mathrm{~kg} \cdot \mathrm{mol}^{-1}$ ); the detailed procedure is described in the Supporting Information. Specifically for this study, the PAA brushes were synthesized on $100 \times 50 \times 10 \mathrm{~mm}^{3}$ silicon single crystals. They remained stable in the $\mathrm{pH}$ conditions at which they were studied. The brush thickness was measured by ellipsometry and the brush chemistry was characterized by infrared spectroscopy in the Attenuated Total Reflection mode. Both thickness and infra-red spectra were identical before and after the experiments.

The synthesis of PNIPAM brushes is somewhat similar to that of PAA brushes, except that carboxy-terminated poly(N-isopropylacrylamide) chains $\left(M=121 \mathrm{~kg} \cdot \mathrm{mol}^{-1}\right)$ were thermally reacted on the GPS-functionalized single crystals at $150{ }^{\circ} \mathrm{C}$. The substrates were used after being extensively rinsed with water and dried at room temperature under a nitrogen flux.

\subsection{Synthesis of hydrogels}

The synthesis of poly(acrylamide) or poly $(N, N$-dimethylacrylamide) hydrogels was described in detail elsewhere. ${ }^{30}$ Free radical polymerization was carried out using $N, N$ '-methylene-bisacrylamide (MBA) as a cross-linker and the potassium persulfate/tetramethylethylenediamine (KPS/TEMED) redox couple as initiator. Unless otherwise noted, gels were synthesized with 
a monomer to deuterium oxide $\left(\mathrm{D}_{2} \mathrm{O}\right)$ mass ratio of $10 \%$, an MBA to monomer molar ratio of $2 \%$ and an initiator to monomer molar ratio of $1 \%$. Two hydrophobic glass slides (treated by octadecyltrimethoxysilane) sandwiched a PDMS gasket to be used as molds for the formation of hydrogels. By taking the glass slides apart from the gasket, the PAM or PDMA gels were removed from the glass mold; they were dialyzed in $\mathrm{D}_{2} \mathrm{O}$ and were finally stored in their swollen state until final use. The hydrophobic treatment allows the demolding of the gel from the glass molds without causing any damage to the surfaces of the gels. The gels were then put in contact with the PAA brushes on the silicon single crystals.

\subsection{Neutron reflectivity}

Neutron reflectivity measurements were carried out on the time-of-flight reflectometer EROS at the Laboratoire Léon Brillouin, CEA-Saclay (France). On this reflectometer equipped with a ${ }^{3} \mathrm{He}$ detector, the accessible wavelengths are in the range of 3 to $25 \AA(\Delta \lambda / \lambda \approx 0.1)$, the sample-to-detector distance is fixed at $2.0 \mathrm{~m}$ and we used a grazing angle of $1.34^{\circ}$, giving access to a wave vector range of $5.810^{-3} \AA^{-1}-4.910^{-2} \AA^{-1}$. The acquisition time was set between 6 and 8 hours during which the samples were regulated at $25^{\circ} \mathrm{C}$ for the PAA brushes and either $20^{\circ} \mathrm{C}$ or $60{ }^{\circ} \mathrm{C}$ for the PNIPAM brushes. The beam size was adapted to the sample size and incident angle: $25 \mathrm{~mm}$ wide and $1 \mathrm{~mm}$ high. A schematic of the sample cell is shown in the SI: it consists of the silicon single crystal and a thermoregulated Teflon trough (in which the deuterium oxide or the swollen gels are placed) sandwiched in a stainless steel holder.

The reflectivity raw data was corrected for the direct beam and for the non-specular signal; it was then normalized using the position of the total reflectivity plateau. The logarithm of the

corrected intensity is plotted as a function of the wave vector $q=\frac{2 \pi}{\lambda} \sin (\theta)$, where $\lambda$ is the wavelength and $\theta$ the grazing angle. 
Neutron reflectivity is sensitive to the scattering length density profile normal to the interface $N b(z)$ which provides the monomer volume fraction profile $\phi(z)$ or the local monomer concentration at a distance $z$ from the interface. The reflectivity experiments were performed using in all cases hydrogenated polymer brushes and deuterium oxide. The choice of a hydrogenated polymer brush in a deuterated solvent gave access to a total reflection plateau and a low incoherent neutron scattering. However two types of experiments were carried out; in experiment 1 , the brush was immersed in pure $\mathrm{D}_{2} \mathrm{O}$, while in experiment 2 the brush was in intimate contact with a gel (hydrogenated PAM or PDMA) swollen in $\mathrm{D}_{2} \mathrm{O}$. In the data analysis, we assume that (i) the PAM or PDMA networks are continuous media - which basically means that the concentration of the networks is independent of the distance from the surface within the penetration depth of the neutrons; and (ii) the scattering signal from the hydrogenated polymer in the swollen gel has a negligible contribution to the reflectivity signal. The extents to which these assumptions are valid are discussed in the next section.

The density profile deduced from the neutron reflectivity data is the profile of the polymer brush either in $\mathrm{D}_{2} \mathrm{O}$ or in contact with the $\mathrm{D}_{2} \mathrm{O}$-swollen hydrogel. The scattering length densities used for the determination of the profile of the brush were $0.7810^{-6} \AA^{-2}$ for PNIPAM, $2.6810^{-6} \AA^{-2}$ for neutral $d_{1}$-PAA (PAA in $\mathrm{D}_{2} \mathrm{O}$ ) and $2.1010^{-6} \AA^{-2}$ for ionized PAA, $2.0710^{-6} \AA^{-2}$ for silicon, $3.4810^{-6} \AA^{-2}$ for silica and GPS, and $(6.25 \pm 0.15) 10^{-6} \AA^{-2}$ for the $\mathrm{pH}$ equilibrated deuterated water or hydrogels. The brush was modeled as a set of layers, each characterized by a fixed thickness and a fixed scattering length density. Two adjacent layers were connected using error functions (erf) of fixed width to get a continuous profile. The procedure consisted of choosing a scattering length density profile and finding the corresponding parameters for which the calculated reflectivity curve fitted the experimental reflectivity data the best. This reliable method allowed the determination of a continuous 
scattering length density profile, without making any assumptions about its analytical form. The monomer volume fraction profile $\phi(z)$ was then deduced from $N b(z) .{ }^{31}$

From the density profile $\phi(z)$, one can calculate the dry thickness $\gamma$ of the polymer brush: $\gamma=\int_{0}^{+\infty} \phi(z) d z \cdot \gamma$ is independent of the shape of $\phi(z)$ and can be compared to the values provided by ellipsometry: in all cases, the thickness measured by ellipsometry was identical to the thickness obtained from simulated density profiles. The swollen thickness of the brush $L$ can also be calculated from the profile $\phi(z): L=2 \frac{\int_{0}^{+\infty} z \cdot \phi(z) \cdot d z}{\int_{0}^{+\infty} \phi(z) \cdot d z}$. It is twice the normalized first moment of the volume fraction profile which is defined with complementary error functions (erf) connecting two adjacent layers. The degree of swelling defined as the ratio of the thickness of the swollen brush to that of the dry brush, $L / \gamma$, quantifies the level of stretching of the chains. Similarly to the swollen thickness, the mean volume fraction $\bar{\phi}$ can be calculated from the profile: $\bar{\phi}=2 \frac{\int_{0}^{+\infty} \phi \cdot z(\phi) \cdot d \phi}{\int_{0}^{+\infty} z(\phi) \cdot d \phi}$. 


\section{RESULTS AND DISCUSSION}

Before presenting the main results of the study we need to take a critical look at the assumption that the reflectivity signal and analysis can be treated as if the PAM or PDMA networks were continuous mediums, which basically means that their concentration is independent of the distance from the surface.

\subsection{Effect of the presence of hydrogels on the determination of the brush density profile}

3.1.1 The gel concentration at its surface is low. In a previous paper, we have shown that the position of the critical wave vector of the total reflection plateau $\left(q_{\text {crit }}\right)$ can be used to determine the average hydrogel concentration at the interface between the deuterated water and the silicon wafer. ${ }^{30}$ Using this method, we demonstrated that when a PDMA hydrogel (DMA to water mass ratio of $10 \mathrm{wt} \%$ and MBA to DMA molar ratio of $2 \mathrm{~mol} \%$ ) is synthesized between OTS-treated glass plates and put in $\mathrm{D}_{2} \mathrm{O}$ to reach its equilibrium swelling, the polymer volume fraction at the surface $\left(\varphi_{\text {surf }}=410^{-3}\right)$ is significantly lower than the polymer concentration in the bulk of the gel $\left(\varphi_{\text {bulk }}=7.110^{-2}\right)$. This means that for our system, the signal from the hydrogenated PAA grafted brush (of maximum scattering length density $2.6810^{-6} \AA^{-2}$ for neutral PAA), the concentration of which can reach tens of percent at the surface, is significantly higher than the signal due to the gel networks, the SLD of which are either $0.9410^{-6} \AA^{-2}$ for PDMA or $3.1610^{-6} \AA^{-2}$ for PAM. Except in the region at the very top surface of the brush, where the volume fraction of PAA becomes smaller than a few percents, it is reasonable to consider that the reflectivity signal coming from the gel is negligible. The whole reflectivity signal on the first $500 \mathrm{~nm}$ near the surface is dominated by the polymer brush. ${ }^{32}$ 


\subsubsection{The PNIPAM brush, as a control sample, demonstrating that the gel does not}

invalidate the reflectivity analysis. In this part, we use a control sample made of PNIPAM (SLD $\sim 0.7510^{-6} \AA^{-2}$ ) brushes to check the influence of the gel on the determination of the structure of the brush. PAM (from the gel) and PNIPAM (from the brush) are known not to form complexes in water at any $\mathrm{pH}$ or temperature conditions, but PNIPAM is a well studied thermo-sensitive polymer for its LCST in water at $32{ }^{\circ} \mathrm{C}$. Thus, a significant difference in the conformation of the brush is expected for the PNIPAM brush immersed in water when changing the temperature from $20^{\circ} \mathrm{C}$ to $60^{\circ} \mathrm{C}$. Using the setup shown in the Supporting Information, we have measured the reflectivity of a PNIPAM brush at $\mathrm{T}=20{ }^{\circ} \mathrm{C}<\mathrm{LCST}$ when the polymer is soluble in $\mathrm{D}_{2} \mathrm{O}$ and at $\mathrm{T}=60^{\circ} \mathrm{C}>\mathrm{LCST}$ when the polymer is collapsed. These measurements have been carried out in pure $\mathrm{D}_{2} \mathrm{O}$ and in contact with a PAM hydrogel equilibrated at $\mathrm{pH} 9$ in $\mathrm{D}_{2} \mathrm{O}$.

The reflectivity curves obtained in water at $20^{\circ} \mathrm{C}$ and at $60{ }^{\circ} \mathrm{C}$ are shown on Figure 1 . Comparing these curves, one can first notice the difference in the Kiessig fringes, which are more pronounced at $60{ }^{\circ} \mathrm{C}$ and which are the signature of a steeper density profile. At $20^{\circ} \mathrm{C}$, the brush is stretched away from the surface with a maximum extension of $1400 \AA$ (about five times further from the surface than the PAA brushes studied later). On the contrary at 60 ${ }^{\circ} \mathrm{C}$, the brush is collapsed on the surface (maximum extension below $400 \AA$ ) with a small depleted layer and a high polymer fraction $(\sim 0.6)$ close to the surface. The LCST has caused the appearance of two phases: polymer-poor and polymer-rich. Nevertheless, the PNIPAM chains are not completely collapsed on the surface since the polymer profile is not that of a dry brush. 

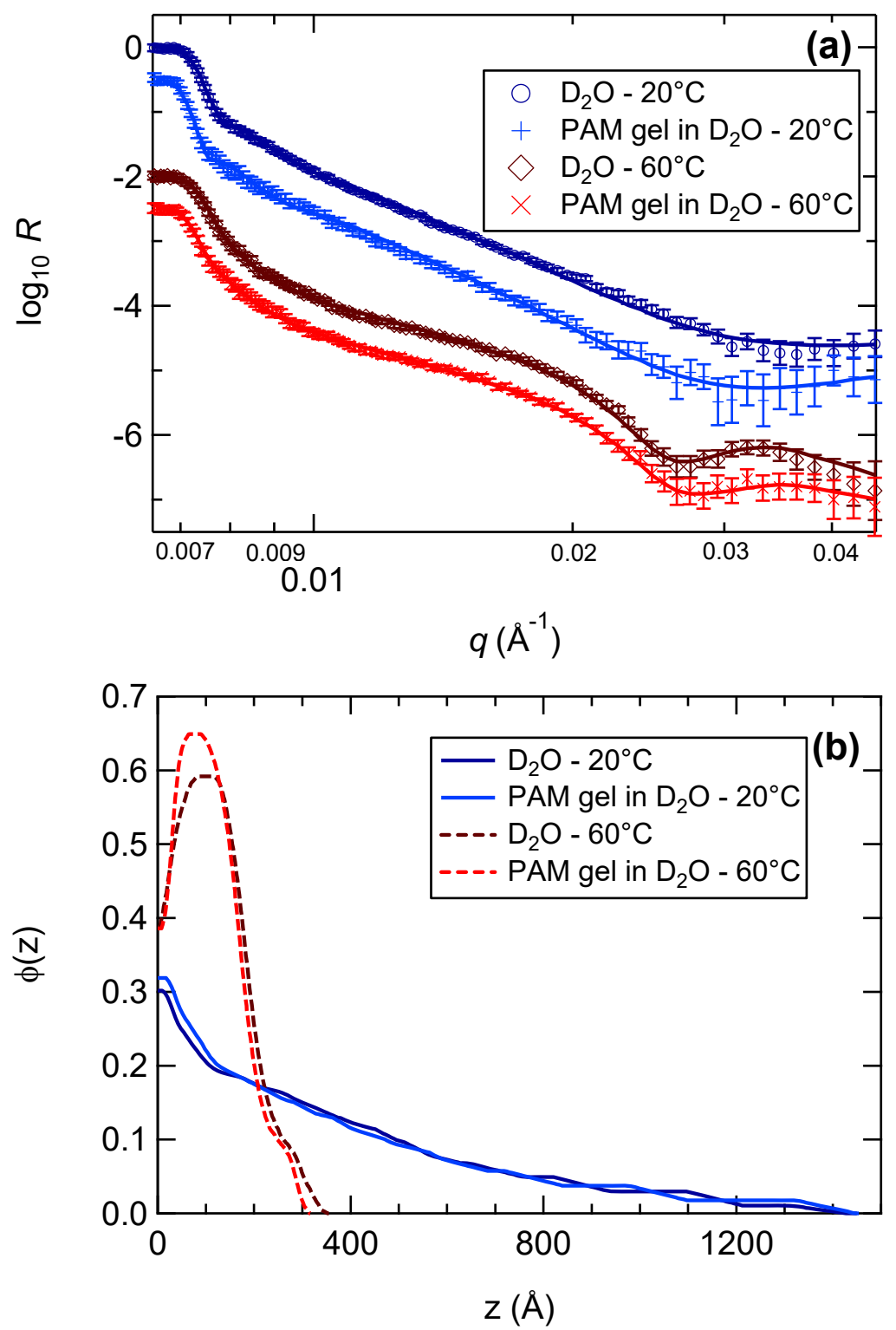

Figure 1. (a) Neutron reflectivity curves and (b) polymer volume fraction corresponding to the best fit of the reflectivity data for temperature-responsive PNIPAM brushes. The reflectivity curves are shifted vertically for clarity. In figure (a), the immersing medium is, from top to bottom: $\mathrm{D}_{2} \mathrm{O}$ at $20{ }^{\circ} \mathrm{C}$; PAM gel in $\mathrm{D}_{2} \mathrm{O}$ at $20{ }^{\circ} \mathrm{C} ; \mathrm{D}_{2} \mathrm{O}$ at $60{ }^{\circ} \mathrm{C}$; PAM gel in $\mathrm{D}_{2} \mathrm{O}$ at $60^{\circ} \mathrm{C}$. In figure (b), solid lines are for the brushes immersed at $20^{\circ} \mathrm{C}$ and dashed lines for the brushes immersed at $60^{\circ} \mathrm{C}$; dark colors are for brushes immersed in $\mathrm{D}_{2} \mathrm{O}$, light colors for the brushes in contact with the $\mathrm{D}_{2} \mathrm{O}$-swollen PAM gel. 
In the presence of the PAM gel, the profiles of the PNIPAM brushes obtained from the fits (which neglect the presence of the gel) are nearly identical; a small difference can actually be seen close to the total reflection plateau (see Figure 1). This result confirms that the reflectivity signal is dominated by the signal from the brush and that the presence of the gel is only visible through a slight modification of the total reflection plateau. In other words, our hypothesis (considering the gels as continuous semi-infinite mediums, $c f$. Neutron reflectivity section in the experimental part) is valid.

In conclusion, three main reasons justify neglecting the gel in determining the brush profile: (a) First, we have shown that the gel concentration at its surface is a lot smaller than the concentration in the bulk of the gel, and as a consequence, it is possible to consider that within the neutron penetration depth in the gel (which is about $500 \mathrm{~nm}$ ), the gel concentration remains low $\left(\varphi \approx 410^{-3}\right)$ and constant or its variation is small close to the surface.

(b) Secondly, we have used a control system where the brush is made of PNIPAM, which has no specific interaction with the gels, to show that the presence of the gel was not invalidating the analysis method of the neutron reflectivity signal.

(c) Finally in the absence of interactions at $\mathrm{pH} \mathrm{9,} \mathrm{the} \mathrm{determined} \mathrm{profile} \mathrm{of} \mathrm{the} \mathrm{PAA} \mathrm{brush} \mathrm{is}$ identical in water or in contact with the gel, as could be expected (see Figure 2); this latter result also demonstrates that the scattering signal from the gel has a negligible contribution to the reflectivity signal.

\subsection{Effect of pH on the stretching of the brush in contact with the gel}

Figure 2 displays the neutron reflectivity curves and the corresponding density profiles of the polymer brush which best fit the experimental curves; the data are shown for the PAA brush 
immersed in $\mathrm{D}_{2} \mathrm{O}$ and in contact with the PAM hydrogel at $\mathrm{pH}$ 9. The curves show no obvious Kiessig fringes, suggesting that the density profiles are not sharp. In $\mathrm{D}_{2} \mathrm{O}$ at $\mathrm{pH}$, the PAA brush is completely ionized and stretched. The density profile has a Gaussian shape, as expected from theoretical models. ${ }^{33,34}$ The reflectivity data do not change much, whether the brush is immersed in $\mathrm{D}_{2} \mathrm{O}$ or in contact with the gel and therefore the density profiles of the brush obtained at $\mathrm{pH} 9$ are very close. This is expected since no specific interaction is expected to occur at high $\mathrm{pH}$ between the PAA chains of the brush and the PDMA or PAM chains of the hydrogel. ${ }^{27}$
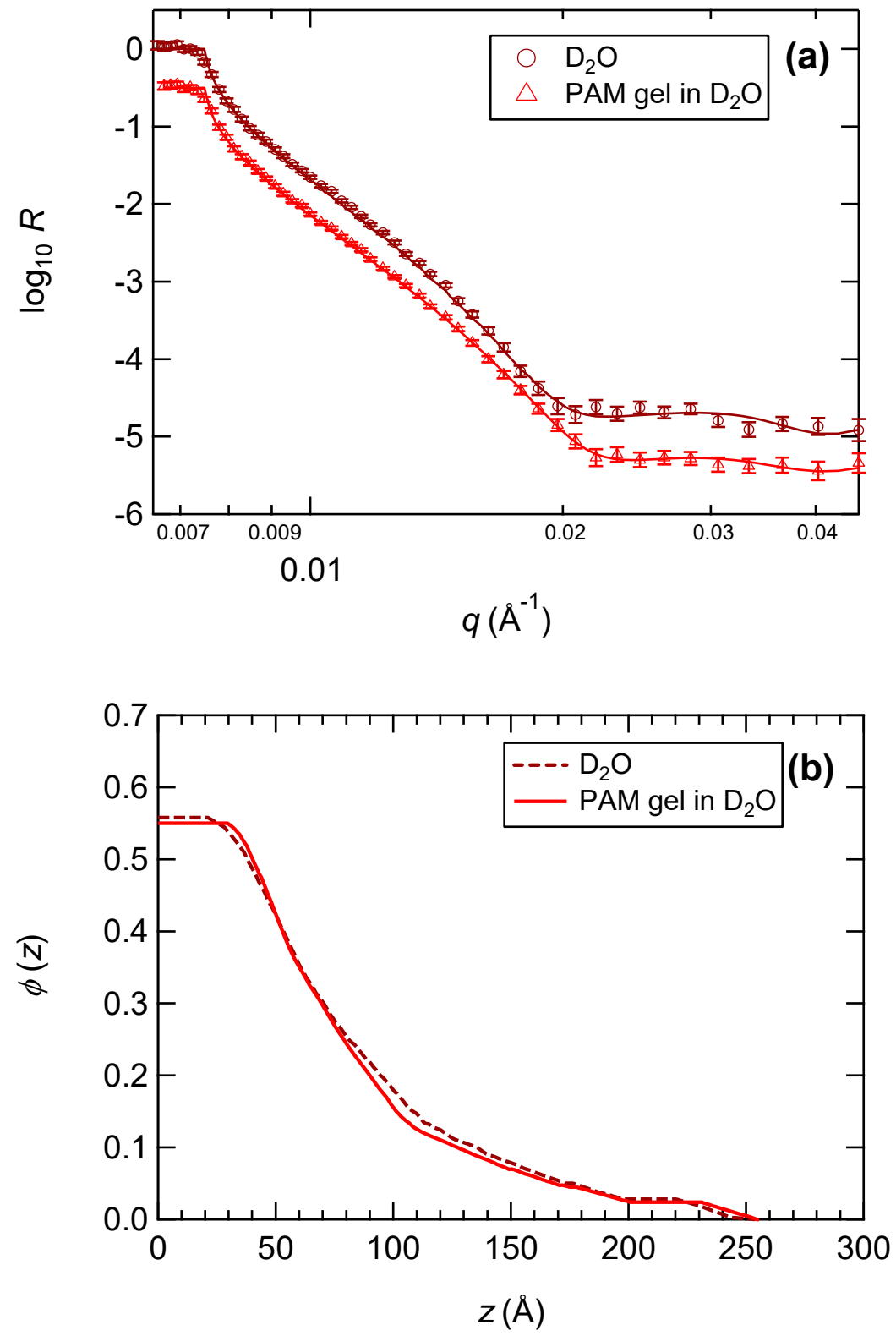
Figure 2. (a) Neutron reflectivity curves and (b) polymer volume fraction profiles corresponding to the best fit of the reflectivity data. Experiments were carried out with the PAA brush at $\mathrm{pH} 9$, in $\mathrm{D}_{2} \mathrm{O}$ (dark red and dashed line) or in contact with a PAM gel (light red and solid line). The reflectivity curves are shifted vertically for clarity.

In Figure 3, the results at $\mathrm{pH} 2$ are shown. As observed at $\mathrm{pH} 9$, the density profiles are very gradual. At $\mathrm{pH} 2$, the brush in contact with $\mathrm{D}_{2} \mathrm{O}$ has a parabolic profile, as expected by theoretical models. ${ }^{35-37}$ However, when the brush is in contact with the gel, it is obvious that it is more stretched. At $\mathrm{pH} \mathrm{2,} \mathrm{the} \mathrm{PAA} \mathrm{chains} \mathrm{are} \mathrm{protonated} \mathrm{and} \mathrm{not} \mathrm{ionized} \mathrm{so} \mathrm{that} \mathrm{PAA} \mathrm{and}$ PAM (or PDMA) chains can form interpolymer complexes through hydrogen bonds. ${ }^{27}$ The specific interactions through hydrogen bonds between the brush and the gel are the most likely reason for the stretching of the brush.

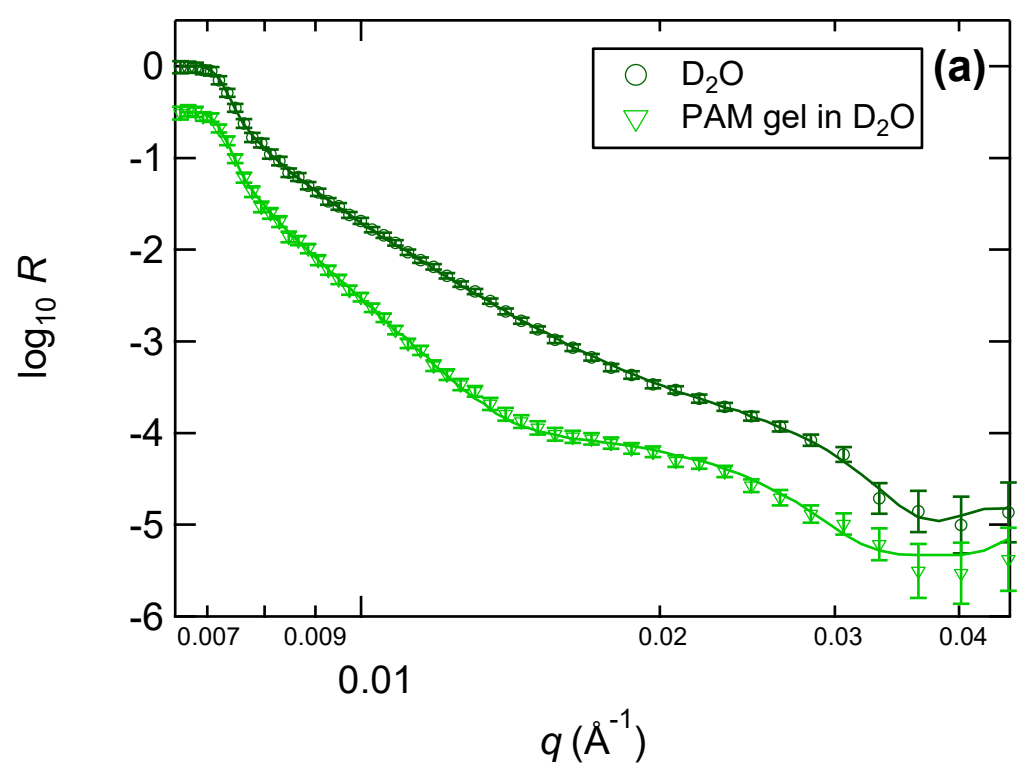




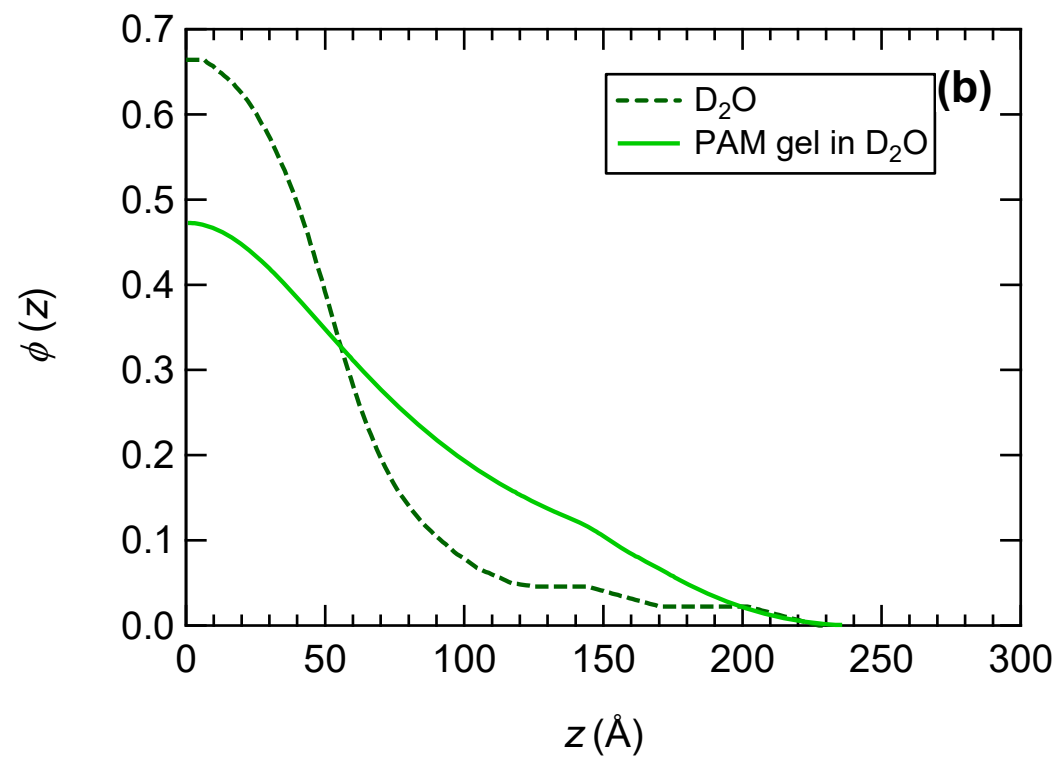

Figure 3. (a) Neutron reflectivity curves and (b) polymer volume fraction profiles corresponding to the best fit of the reflectivity data. Experiments are carried out with the PAA brush at $\mathrm{pH} 2$, in $\mathrm{D}_{2} \mathrm{O}$ (dark green and dashed line) or in contact with a PAM gel (light green and solid line). The reflectivity curves are shifted vertically for clarity.

The mean characteristics of the brushes (the dry thickness $\gamma$, the mean height in the swollen state $L$, the mean volume fraction of polymer inside the brush $\bar{\phi}$ and the swelling ratio $L / \gamma$ ) calculated from the profiles are displayed in Table 1 . The swelling ratio at $\mathrm{pH} 2$ in the presence of the gel is even higher than that of the brush at $\mathrm{pH} 9$. For many different experiments carried out at $\mathrm{pH} 2$, we systematically found that the mean height of the brush was extended of at least $20 \%$ when in contact with the gel. The same applies to the swelling ratio of the brush which was in the range of (or slightly higher than) the swelling ratio obtained at $\mathrm{pH} 9$. Accordingly, the mean polymer volume fraction is smaller for more stretched brushes (at $\mathrm{pH} 9$ and in contact with the gel at $\mathrm{pH}$ 2) for the same dry thickness or integral of the profile. These results suggest that the H-bonds are the source of the stretching of the brush in contact with the gel. This additional extension could be explained by the interpenetration of the brush inside the gel. This effect differs from what was observed for a 
gel and a brush interacting through Coulombic interactions by La Spina et al. ${ }^{10}$ They took advantage of the $\mathrm{pH}$-sensitive electrostatic complexation between a weak polyacid gel (precisely poly(methacrylic acid) PMMA) and a weak polybase brush (poly[2-(dimethyl amino)ethyl methacrylate] PDMAEMA) to tune the adhesive properties. The two neutron reflectivity curves shown in their work were obtained by using a deuterated PDMAEMA brush in $\mathrm{H}_{2} \mathrm{O}$ at $\mathrm{pH} 7$ and in contact with a hydrogenated PMAA hydrogel. The density profiles revealed that the brush was not extended at its extremity but rather had a sharp interface with the gel, suggesting that there was no interpenetration of the brush inside the gel. In their system, both the PDMAEMA brush and the PMAA hydrogel are polyelectrolytes at $\mathrm{pH} 7$ whereas our system is made of neutral chains at $\mathrm{pH} 2$ : the osmotic pressure due to the presence of counterions could explain the difference between the two systems. Additionally, H-bonds are very short and oriented interactions, which can be formed only when the interacting molecules are in very close vicinity, while Coulombic interactions are active on longer distances.

\begin{tabular}{|c|c|c|c|c|}
\hline Immersing medium & $\gamma(\AA)$ & $L(\AA)$ & $\bar{\phi}$ & $L / \gamma$ \\
\hline $\mathrm{D}_{2} \mathrm{O}, \mathrm{pH} 9$ & 43 & 116 & 0.36 & 2.77 \\
\hline PAM gel, $\mathrm{pH} 9$ & 43 & 116 & 0.36 & 2.77 \\
\hline $\mathrm{D}_{2} \mathrm{O}, \mathrm{pH} 2$ & 43 & 89 & 0.48 & 2.07 \\
\hline PAM gel, $\mathrm{pH} 2$ & 46 & 132 & 0.34 & 2.87 \\
\hline
\end{tabular}

Table 1. Characteristics of swollen poly(acrylic acid) brushes (with $M_{n}=23.6 \mathrm{~kg} / \mathrm{mol}$ ) when varying the immersing medium. The dry thickness $\gamma$, the mean height $L$, the mean volume fraction of polymer inside the brush $\bar{\phi}$ and the swelling ratio $L / \gamma$ are calculated from the profile.

Effect of the characteristics of the gel and of the brush 
Given that the interactions in water for the polymer couples PDMA/PAA and PAM/PAA are different $^{27}$, is it possible to notice a variation in the profile of the brush when the gel chemistry or composition are changed? To answer this question, we have carried out neutron reflectivity measurements on similar but different brushes. We have compared the profile of the brush at $\mathrm{pH} 2$ when it is in contact with pure $\mathrm{D}_{2} \mathrm{O}$ with the profile of the same brush in contact with a swollen gel, either PDMA or PAM. The average characteristics of the brushes as deduced from the measured concentration profiles are given in the Supporting Information. In contact with the PDMA gel, the brush extends further from the surface than in $\mathrm{D}_{2} \mathrm{O}$ and consequently, both the mean height of the brush and its swelling ratio increase. The presence of either gel - PAM or PDMA - stretches similarly the concentration profile of the brush by more than $25 \%$. We also have studied the effect of the gel concentration on the structure of the brush by using PAM hydrogels synthesized with 5, 10 and 15 wt $\%$ of monomer in water (results given in Supporting Information). When varying the gel concentration, the distance between anchoring sites $D$ (specific lengthscale of the brush) was kept constant at $28 \AA$ and the specific length scale of the gel $\xi$ (the thermal correlation length) did not vary much (between 10 and $13 \AA$ as described in detail in the Supporting Information). No trend could be observed.

Besides changing the gel concentration, the number of possible interactions between the gel and the brush and the ratio of the specific length scales can also be varied by changing the grafting density of the brush or the length of the chains. In the Supporting Information, we report the average characteristics of the brush conformations for various $D$ ( $27 \AA$ to $37 \AA)$ and chain lengths $\left(\mathrm{M}=23.6\right.$ or $\left.2.36 \mathrm{~kg} \cdot \mathrm{mol}^{-1}\right)$ in contact with PDMA hydrogels $(\xi \approx 25 \AA)$ : all chains are extended at $\mathrm{pH} 2$ in the presence of the gel but no obvious effect of the grafting density could be observed within the range that we studied. 


\section{Relationship between microscopic structure and macroscopic measurements}

In terms of macroscopic measurements, it is not easy to measure the energy of formation of the polymer complexes in water by micro-differential scanning calorimetry. ${ }^{27}$ However, the macroscopic adhesion has been found larger for PAM hydrogels than for PDMA hydrogels: at $\mathrm{pH} 2$ and for a contact time of $300 \mathrm{~s}$, the energy of adhesion for 2 similar PAA brushes is of about $10 \mathrm{~mJ} . \mathrm{m}^{-2}$ for a PDMA hydrogel and of about $100 \mathrm{~mJ} . \mathrm{m}^{-2}$ for a PAM hydrogel (both synthesized with $10 \mathrm{wt} \%$ of monomers in water and 2 mol $\%$ of crosslinker). ${ }^{26}$ In terms of interactions of entropic origin, the additional stretching of the brush for the PAM gel compared to the PDMA gel is not sufficient to account for the difference in adhesion energies measured.

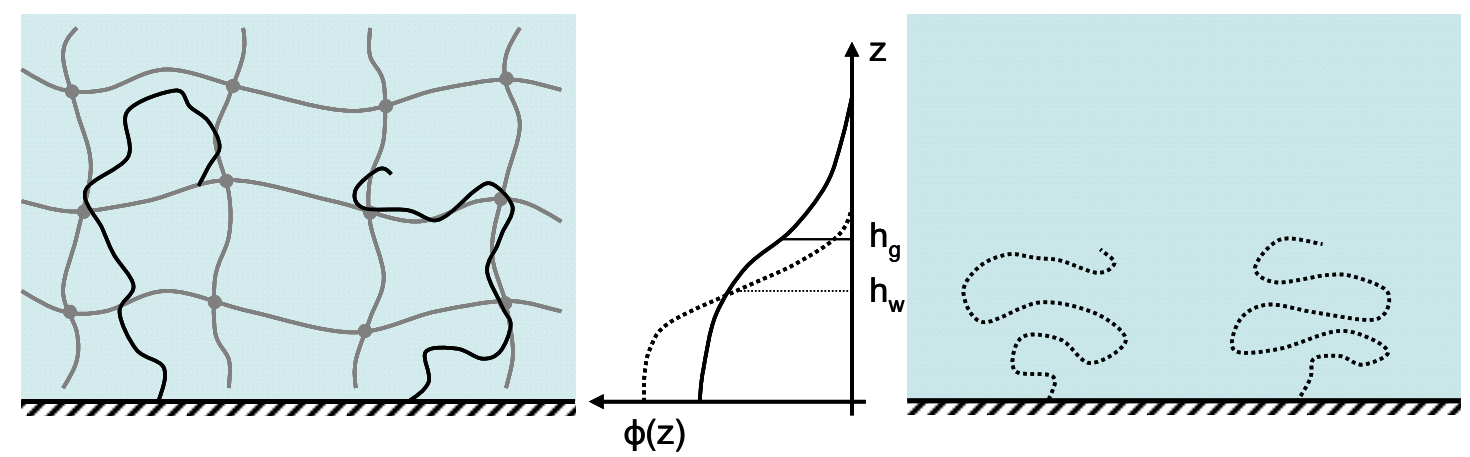

Figure 4. Schematic representation of the brush structure against the gel and against water at $\mathrm{pH}$ 2. The brush is more extended in the presence of the gel at $\mathrm{pH} 2$ when there are specific interactions between the gel and the brush with hydrogen bonds. $h_{g}$ and $h_{w}$ are the mean heights of the brush in the presence of the gel, and in water respectively.

If the system is in thermodynamic equilibrium, the loss in free energy due to the additional stretching of the brushes induced by the presence of the gel, as schematized on Figure 4, might be a way to measure or characterize the energy of the interactions. This elastic energy can be statistically calculated using a Flory-type self-consistent approach: 


$$
F_{e l}(k T / \text { chain })=\frac{h_{g}^{2}-h_{w}^{2}}{R_{0}^{2}}
$$

By using this expression with the value of $R_{0}=\left(C_{\infty} n l^{2} / 6\right)^{1 / 2} \approx 35 \AA$ (with $n=330$, $l=1.54 \AA$ and $C_{\infty} \approx 8.3$ ), we obtain an additional elastic energy in the range of 1.5 $8 \mathrm{kT} /$ chain. $^{38}$ This is much smaller than the in-air thermodynamic work of adhesion and it is also far below the energy of adhesion measured under water with the PDMA hydrogel (synthesized with a monomer to mass ratio of $10 \%$ and a cross-linker to monomer molar ratio of $2 \%$ ), which is the weakest and of the order of $50 \mathrm{kT} / \mathrm{chain}$. It is more likely that the additional stretching observed favors the formation of longer lived multiple hydrogen bonds which should have an important effect on macroscopic adhesion. 


\section{CONCLUSION}

This work was motivated by understanding the effects of tunable H-bond interactions on the conformation of a $\mathrm{pH}$-sensitive brush in contact with a hydrogel. Complementary to the macroscopic approach which consisted in underwater adhesion test, ${ }^{26}$ this paper explores the structural aspects of these interactions at the molecular scale by using neutron reflectivity. After demonstrating that the presence of a gel as a semi-infinite medium does not alter the reflectivity study, the structures of the polymer brush were determined in contact with either water or a hydrogel, both of them being equilibrated at the same $\mathrm{pH}$.

pH-responsive PAA brushes were synthesized on silicon wafers following the "grafting onto" approach. They form hydrogen bonds triggered by $\mathrm{pH}$ with chemically cross-linked model hydrogels of PAM and PDMA. As a result, we have shown that at $\mathrm{pH} 2$ (when the brushes are able to form H-bonds with the gels), the brushes are additionally stretched when they are in contact with the swollen gel instead of being immersed in $\mathrm{D}_{2} \mathrm{O}$, indicating that the presence of polymer-polymer interactions has an effect on the conformation of the brush. We found the same additional stretching of the brush for both PAM and PDMA hydrogels at any concentration between 5 and 15 wt \% (of monomers in the gel) and for various grafting densities of the polymer brush. The effect of the additional stretching of the brush was attenuated for short chains for which the number of hydrogen bonds per chain causing the interpolymer complexes with the hydrogel was weaker. 


\section{ACKNOWLEDGMENTS}

We gratefully thank the French National Research Agency (ANR) for its financial support.

\section{SUPPORTING INFORMATION AVAILABLE}

In Supporting Information are provided the full procedure for the PAA brushes synthesis, an extensive discussion of the neutron reflectivity technique in the configuration of our samples, and the characterization of the thermal correlation lengths by Small Angle Neutron Scattering in our hydrogel samples. This information is available free of charge via the Internet at http://pubs.acs.org/. 


\section{REFERENCES}

1. Peppas, N. A.; Sahlin, J. J., Hydrogels as mucoadhesive and bioadhesive materials: A review. Biomaterials 1996, 17, (16), 1553-1561.

2. Drury, J. L.; Mooney, D. J., Hydrogels for tissue engineering: scaffold design variables and applications. Biomaterials 2003, 24, (24), 4337-4351.

3. Leung, S. H. S.; Robinson, J. R., Bioadhesive Drug Delivery. ACS Symp. Ser. 1991, 467, 350366.

4. Hägerström, H.; Bergström, C. A. S.; Edsman, K., The importance of gel properties for mucoadhesion measurements: a multivariate data analysis approach. J. Pharm. Pharmacol. 2004, 56, (2), 161-168.

5. Shoichet, M. S., Polymer Scaffolds for Biomaterials Applications. Macromolecules 2010, 43, (2), 581-591.

6. Gong, J. P., Friction and lubrication of hydrogels - its richness and complexity. Soft Matter 2006, 2, (7), 544-552.

7. Yashima, S.; Takase, N.; Kurokawa, T.; Gong, J. P., Friction of hydrogels with controlled surface roughness on solid flat substrates. Soft Matter 2014, 10, (18), 3192-3199.

8. Rose, S.; Prevoteau, A.; Elziere, P.; Hourdet, D.; Marcellan, A.; Leibler, L., Nanoparticle solutions as adhesives for gels and biological tissues. Nature 2014, 505, (7483), 382-385.

9. Huang, Y. B.; Szleifer, I.; Peppas, N. A., Gel-gel adhesion by tethered polymers. J. Chem. Phys. 2001, 114, (8), 3809-3816.

10. La Spina, R.; Tomlinson, M. R.; Ruiz-Perez, L.; Chiche, A.; Langridge, S.; Geoghegan, M., Controlling network-brush interactions to achieve switchable adhesion. Angew. Chem., Int. Ed. 2007, 46, (34), 6460-6463.

11. Advincula, R. C.; Brittain, W. J.; Caster, K. C.; Rühe, J., Polymer Brushes. Wiley-VCH: Weinheim (Germany), 2004.

12. Currie, E. P. K.; Norde, W.; Stuart, M. A. C., Tethered polymer chains: surface chemistry and their impact on colloidal and surface properties. Adv. Colloid Interface Sci. 2003, 100, 205-265.

13. Zdyrko, B.; Klep, V.; Li, X. W.; Kang, Q.; Minko, S.; Wen, X. J.; Luzinov, I., Polymer brushes as active nanolayers for tunable bacteria adhesion. Mater. Sci. Eng., C 2009, 29, (3), 680-684.

14. Collett, J.; Crawford, A.; Hatton, P. V.; Geoghegan, M.; Rimmer, S., Thermally responsive polymeric hydrogel brushes: synthesis, physical properties and use for the culture of chondrocytes. $J$. R. Soc., Interface 2007, 4, (12), 117-126.

15. Iwata, R.; Satoh, R.; Iwasaki, Y.; Akiyoshi, K., Covalent immobilization of antibody fragments on well-defined polymer brushes via site-directed method. Colloids Surf., B 2008, 62, (2), 288-298.

16. Wang, Y.-M.; Cui, Y.; Cheng, Z.-Q.; Song, L.-S.; Wang, Z.-Y.; Han, B.-H.; Zhu, J.-S., Poly(acrylic acid) brushes pattern as a 3D functional biosensor surface for microchips. Appl. Surf. Sci. 2013, 266, (0), 313-318.

17. Moglianetti, M.; Webster, J. R. P.; Edmondson, S.; Armes, S. P.; Titmuss, S., A Neutron Reflectivity Study of Surfactant Self-Assembly in Weak Polyelectrolyte Brushes at the SapphireWater Interface. Langmuir 2011, 27, (8), 4489-4496.

18. Retsch, M.; Walther, A.; Loos, K.; Muller, A. H. E., Synthesis of dense poly(acrylic acid) brushes and their interaction with amine-functional silsesquioxane nanoparticles. Langmuir 2008, 24, (17), 9421-9429.

19. Bosker, W. T. E.; Iakovlev, P. A.; Norde, W.; Cohen Stuart, M. A., BSA adsorption on bimodal PEO brushes. J. Colloid Interface Sci. 2005, 286, (2), 496-503.

20. Evers, F.; Reichhart, C.; Steitz, R.; Tolan, M.; Czeslik, C., Probing adsorption and aggregation of insulin at a poly(acrylic acid) brush. Phys. Chem. Chem. Phys. 2010, 12, (17), 4375-4382.

21. Hollmann, O.; Czeslik, C., Characterization of a planar poly(acrylic acid) brush as a materials coating for controlled protein immobilization. Langmuir 2006, 22, (7), 3300-3305. 
22. Hollmann, O.; Gutberlet, T.; Czeslik, C., Structure and protein binding capacity of a planar PAA brush. Langmuir 2007, 23, (3), 1347-1353.

23. Hollmann, O.; Steitz, R.; Czeslik, C., Structure and dynamics of alpha-lactalbumin adsorbed at a charged brush interface. Phys. Chem. Chem. Phys. 2008, 10, (10), 1448-1456.

24. Unsworth, L. D.; Sheardown, H.; Brash, J. L., Protein-Resistant Poly(ethylene oxide)-Grafted Surfaces: Chain Density-Dependent Multiple Mechanisms of Action. Langmuir 2008, 24, (5), 19241929.

25. Leger, L.; Raphael, E.; Hervet, H., Surface-anchored polymer chains: Their role in adhesion and friction. Adv. Polym. Sci. 1999, 138, 185-225.

26. Sudre, G.; Olanier, L.; Tran, Y.; Hourdet, D.; Creton, C., Reversible adhesion between a hydrogel and a polymer brush. Soft Matter 2012, 8, (31), 8184-8193.

27. Sudre, G.; Tran, Y.; Creton, C.; Hourdet, D., pH/Temperature control of interpolymer complexation between poly(acrylic acid) and weak polybases in aqueous solutions. Polymer 2012, 53, (2), 379-385.

28. Sudre, G.; Siband, E.; Hourdet, D.; Creton, C.; Cousin, F.; Tran, Y., Synthesis and Characterization of Poly(acrylic acid) Brushes: "Grafting-Onto" Route. Macromol. Chem. Phys. 2012, 213, (3), 293-300.

29. Sudre, G.; Hourdet, D.; Creton, C.; Cousin, F.; Tran, Y., pH-Responsive Swelling of Poly(acrylic acid) Brushes Synthesized by the Grafting Onto Route. Macromol. Chem. Phys. 2013, 214, (24), 2882-2890.

30. Sudre, G.; Hourdet, D.; Cousin, F.; Creton, C.; Tran, Y., Structure of Surfaces and Interfaces of Poly(N,N-dimethylacrylamide) Hydrogels. Langmuir 2012, 28, (33), 12282-12287.

31. Tran, Y.; Auroy, P.; Lee, L. T., Determination of the structure of polyelectrolyte brushes. Macromolecules 1999, 32, (26), 8952-8964.

32. Russell, T. P., X-ray and neutron reflectivity for the investigation of polymers. Mater. Sci. Rep. 1990, 5, (4), 171-271.

33. Zhulina, E. B.; Borisov, O. V.; Birshtein, T. M., Structure of Grafted Polyelectrolyte Layer. J. Phys. II 1992, 2, (1), 63-74.

34. Chen, H.; Zajac, R.; Chakrabarti, A., Conformational properties of polyelectrolyte brushes: A Monte Carlo and self-consistent-field study. J. Chem. Phys. 1996, 104, (4), 1579-1588.

35. Milner, S. T.; Witten, T. A.; Cates, M. E., A Parabolic Density Profile for Grafted Polymers. Europhys. Lett. 1988, 5, (5), 413-418.

36. Skvortsov, A. M.; Pavlushkov, I. V.; Gorbunov, A. A.; Zhulina, Y. B.; Borisov, O. V.; Pryamitsyn, V. A., Structure of Dense-Grafted Polymer Monolayers. Vysokomol. Soedin., Ser. A 1988, $30,(8), 1615-1622$.

37. Netz, R. R.; Schick, M., Classical theory of polymer brushes. Europhys. Lett. 1997, 38, (1), $37-42$.

38. Brandrup, J.; Immergut, E. H.; Grulke, E. A.; Kurata, M.; Tsunashima, Y., Polymer Handbook. 4th ed.; Wiley Interscience: New York, 1999; Vol. Viscosity - Molecular Weight Relationships and Unperturbed Dimensions of Linear Chain Molecules. 
TOC GRAPHIC

The brush in contact with the gel is additionnally stretched.

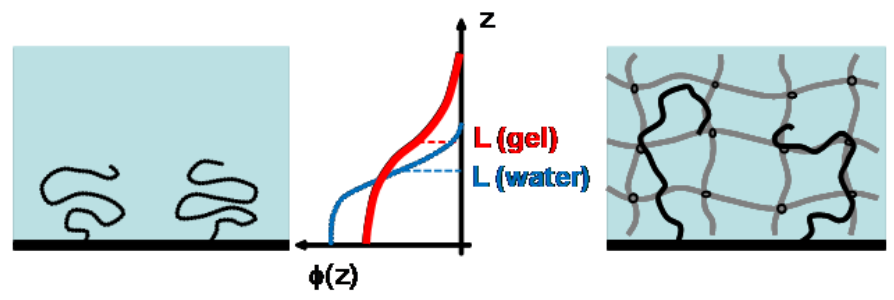

